\title{
THE SLOAN DIGITAL SKY SURVEY QUASAR LENS SEARCH. III. CONSTRAINTS ON DARK ENERGY FROM THE THIRD DATA RELEASE QUASAR LENS CATALOG
}

\author{
Masamune Oguri $^{1,2}$, NaOhisa Inada ${ }^{3,4}$, Michael A. Strauss ${ }^{2}$, Christopher S. KochaneK ${ }^{5}$, Gordon T. Richards ${ }^{6}$, \\ Donald P. Schneider ${ }^{7}$, Robert H. Becker ${ }^{8,9}$, Masataka Fukugita ${ }^{10}$, Michael D. GregG ${ }^{8,9}$, Patrick B. Hall ${ }^{11}$, \\ Joseph F. Hennawi ${ }^{12}$, David E. Johnston ${ }^{13,14}$, Issha Kayo ${ }^{15}$, Charles R. KeEton ${ }^{16}$, Bartosz Pindor ${ }^{17}$, Min-Su Shin ${ }^{2}$, \\ Edwin L. Turner ${ }^{2}$, Richard L. White ${ }^{18}$, Donald G. York $^{19}$, Scott F. Anderson ${ }^{20}$, Neta A. BahCall $^{2}$, \\ Robert J. Brunner ${ }^{21}$, Scott Burles ${ }^{22}$, Francisco J. Castander ${ }^{23}$, Kuenley Chiu ${ }^{24}$, Alejandro Clocchiatti ${ }^{25}$, \\ Daniel Eisenstein ${ }^{26}$, Joshua A. Frieman ${ }^{19,27,28}$, Yozo Kawano ${ }^{15}$, Robert Lupton ${ }^{2}$, Tomoki Morokuma ${ }^{29}$, \\ Hans-Walter Rix ${ }^{30}$, Ryan Scranton ${ }^{31}$, and Erin Scott Sheldon ${ }^{32}$ \\ ${ }^{1}$ Kavli Institute for Particle Astrophysics and Cosmology, Stanford University, 2575 Sand Hill Road, Menlo Park, CA 94025, USA \\ 2 Princeton University Observatory, Peyton Hall, Princeton, NJ 08544, USA \\ ${ }^{3}$ Institute of Astronomy, Faculty of Science, University of Tokyo, 2-21-1 Osawa, Mitaka, Tokyo 181-0015, Japan \\ ${ }^{4}$ Cosmic Radiation Laboratory, RIKEN (The Physical and Chemical Research Organization), 2-1 Hirosawa, Wako, Saitama 351-0198, Japan \\ 5 Department of Astronomy, The Ohio State University, Columbus, OH 43210, USA \\ ${ }^{6}$ Department of Physics, Drexel University, 3141 Chestnut Street, Philadelphia, PA 19104, USA \\ ${ }^{7}$ Department of Astronomy and Astrophysics, The Pennsylvania State University, 525 Davey Laboratory, University Park, PA 16802, USA \\ ${ }^{8}$ IGPP-LLNL, L-413, 7000 East Avenue, Livermore, CA 94550, USA \\ ${ }^{9}$ Department of Physics, University of California at Davis, 1 Shields Avenue, Davis, CA 95616, USA \\ ${ }^{10}$ Institute for Cosmic Ray Research, University of Tokyo, 5-1-5 Kashiwa, Kashiwa City, Chiba 277-8582, Japan \\ ${ }^{11}$ Department of Physics and Astronomy, York University, 4700 Keele Street, Toronto, Ontario, M3J 1P3, Canada \\ 12 Department of Astronomy, University of California at Berkeley, 601 Campbell Hall, Berkeley, CA 94720-3411, USA \\ 13 Jet Propulsion Laboratory, 4800 Oak Grove Drive, Pasadena, CA 91109, USA \\ ${ }^{14}$ California Institute of Technology, 1200 East California Blvd, Pasadena, CA 91125, USA \\ ${ }^{15}$ Department of Physics and Astrophysics, Nagoya University, Chikusa-ku, Nagoya 464-8602, Japan \\ ${ }^{16}$ Department of Physics and Astronomy, Rutgers University, Piscataway, NJ 08854, USA \\ ${ }^{17}$ Space Research Centre, University of Leicester, Leicester LE1 7RH, UK \\ 18 Space Telescope Science Institute, 3700 San Martin Drive, Baltimore, MD 21218, USA \\ ${ }^{19}$ Dept. of Astronomy and Astrophysics, and The Enrico Fermi Institute, 5640 So. Ellis Avenue, The University of Chicago, Chicago, IL 60637, USA \\ ${ }^{20}$ Astronomy Department, Box 351580, University of Washington, Seattle, WA 98195, USA \\ ${ }^{21}$ Department of Astronomy, University of Illinois, 1002 West Green Street, Urbana, IL 61801, USA \\ ${ }^{22}$ Kavli Institute for Astrophysics and Space Research and Department of Physics, Massachusetts Institute of Technology, Cambridge, MA 02139, USA \\ ${ }^{23}$ Institut d'Estudis Espacials de Catalunya/CSIC, Gran Capita 2-4, 08034 Barcelona, Spain \\ ${ }^{24}$ School of Physics, University of Exeter, Stocker Road, Exeter EX4 4QL, UK \\ 25 Departamento de Astronomía y Astrofísica, Pontificia Universidad Católica de Chile, Casilla 306, Santiago 22, Chile \\ ${ }^{26}$ Steward Observatory, University of Arizona, 933 North Cherry Avenue, Tucson, AZ 85721, USA \\ ${ }^{27}$ Kavli Institute for Cosmological Physics, University of Chicago, Chicago, IL 60637, USA \\ ${ }^{28}$ Center for Particle Astrophysics, Fermilab, P.O. Box 500, Batavia, IL 60510, USA \\ ${ }^{29}$ National Astronomical Observatory, 2-21-1 Osawa, Mitaka, Tokyo 181-8588, Japan \\ ${ }^{30}$ Max Planck Institute for Astronomy, Koenigsstuhl 17, 69117 Heidelberg, Germany \\ ${ }^{31}$ University of Pittsburgh, Department of Physics and Astronomy, 3941 O'Hara Street, Pittsburgh, PA 15260, USA \\ 32 Center for Cosmology and Particle Physics, Department of Physics, New York University, 4 Washington Place, New York, NY 10003, USA \\ Received 2007 August 5; accepted 2007 October 29; published 2008 January 15
}

\begin{abstract}
We present cosmological results from the statistics of lensed quasars in the Sloan Digital Sky Survey (SDSS) Quasar Lens Search. By taking proper account of the selection function, we compute the expected number of quasars lensed by early-type galaxies and their image separation distribution assuming a flat universe, which is then compared with seven lenses found in the SDSS Data Release 3 to derive constraints on dark energy under strictly controlled criteria. For a cosmological constant model $(w=-1)$ we obtain $\Omega_{\Lambda}=0.74_{-0.15}^{+0.11}$ (stat. $)_{-0.06}^{+0.13}$ (syst.). Allowing $w$ to be a free parameter we find $\Omega_{\mathrm{M}}=0.26_{-0.06}^{+0.07}$ (stat. $)_{-0.05}^{+0.03}$ (syst.) and $w=-1.1 \pm 0.6$ (stat. $)_{-0.5}^{+0.3}$ (syst.) when combined with the constraint from the measurement of baryon acoustic oscillations in the SDSS luminous red galaxy sample. Our results are in good agreement with earlier lensing constraints obtained using radio lenses, and provide additional confirmation of the presence of dark energy consistent with a cosmological constant, derived independently of type Ia supernovae.
\end{abstract}

Key words: cosmological parameters - cosmology: theory - gravitational lensing

Online-only material: color figure

\section{INTRODUCTION}

The accelerating expansion of the universe is one of the central problems in modern cosmology. This acceleration is usually attributed to the dominant presence of a negativepressure component that is often referred to as dark energy.
There are many models that explain the acceleration, including a classical cosmological constant, decaying scalar fields, and topological defects (e.g., see Peebles \& Ratra 2003, for a review). In addition it might also be explained by long-range modifications of the gravitational force law (e.g., Carroll et al. 2004). 
The dark energy is characterized by its cosmological density $\Omega_{\mathrm{DE}}$, and its equation of state $w$, which is defined as the pressure divided by the density of dark energy. In particular, measuring $w$ is a useful test of models for dark energy. Since $w$ determines the expansion rate of the universe and the cosmological distance to a given redshift, not only $\Omega_{\mathrm{DE}}$ but also the value of $w$ and its time dependence can be inferred from distance (or volume) measurements on cosmological scales. One powerful way to constrain $w$ is via observations of distant type-Ia supernovae that make use of luminosity-decline rate correlations to standardize their luminosities (Riess et al. 1998; Perlmutter et al. 1999): since the standardized luminosities of type-Ia supernovae have small scatter, they serve as an excellent standard candle to measure cosmological distances. Another probe of dark energy is the fluctuation spectrum of the cosmic microwave background (CMB; de Bernardis et al. 2000; Spergel et al. 2003, 2007). The integrated Sachs-Wolfe effect, which can be detected by correlating the CMB map with the large-scale distribution of galaxies, allows a direct detection of the dark energy component (e.g., Rassat et al. 2007). Additional constraints on dark energy come from baryon acoustic oscillations in the galaxy power spectrum (Eisenstein et al. 2005; Cole et al. 2005; Percival et al. 2007) and X-ray clusters of galaxies (Allen et al. 2004; 2007; Rapetti et al. 2005). Since different methods involve different systematics and degeneracies with the cosmological parameters, it is of great importance to use as many independent observations as possible in studying dark energy.

The statistics of strong lensing offer an alternative constraint on dark energy (Turner 1990; Fukugita et al. 1990, but see also Keeton 2002). The probability that a distant object is strongly lensed is proportional to the number of possible lensing objects along the line of sight, and thus quite sensitive to dark energy. This method has been applied to both optical and radio lens samples to derive interesting constraints on the value of the cosmological constant (Maoz \& Rix 1993; Kochanek 1996; Falco et al.1998; Chiba \& Yoshii 1999), but such applications have been limited by the small size of existing lens samples as well as poor knowledge of source and lens populations (Maoz 2005). For instance, past work tended to rule out large values ( $\gtrsim 0.7)$ of $\Omega_{\mathrm{DE}}$ (e.g., Kochanek 1996), because of overestimates of the luminosity function of galaxies (e.g., Chiba \& Yoshii 1999). The most recent lensed quasar survey in the radio band, the Cosmic Lens All-Sky Survey (CLASS; Myers et al. 2003; Browne et al. 2003), contains a statistical sample of 13 lenses. Cosmological constraints from this lens sample are roughly consistent with the current standard model in which the universe is dominated by dark energy (Chae et al. 2002; Chae 2003, 2007; Mitchell et al. 2005).

The Sloan Digital Sky Survey Quasar Lens Search (SQLS; Oguri et al. 2006) provides a large statistical lens sample appropriate for studying cosmology. It is based on the optical quasar catalog from the Sloan Digital Sky Survey (SDSS; York et al. 2000), and therefore is complementary to the CLASS in several ways. In particular the well-known redshift distribution of quasars and the lensing selection function (Oguri et al. 2006, hereafter Paper I) allow an accurate estimate of lensing rates. We present our first complete lens sample from Data Release 3 (DR3; Abazajian et al. 2005; Schneider et al. 2005) in Inada et al. (2008, hereafter Paper II): it consists of 11 lensed quasars with flux ratios of faint to bright images greater than $10^{-0.5}$ (for double lenses) and image separations between $1^{\prime \prime}$ and $20^{\prime \prime}$, selected from 22,683 low-redshift $(0.6<z<2.2)$ quasars brighter than $i=19.1$. In this paper, we use a subsample of this optical lens sample to constrain cosmological parameters, in particular the dark energy abundance and equation of state.

This paper is organized as follows. In Section 2 we briefly summarize our statistical lens sample. Section 3 describes how the expected number of lensed quasars is computed. We present our results in Section 4, and summarize in Section 5. We denote the present matter density as $\Omega_{M}$. The present dark energy density is described as $\Omega_{\mathrm{DE}}$, or $\Omega_{\Lambda}$ if the cosmological constant $w=-1$ is assumed. We use the Hubble constant in dimensionless form $h=H_{0} /\left(100 \mathrm{~km} \mathrm{~s}^{-1} \mathrm{Mpc}^{-1}\right)$. Throughout the paper we assume a flat universe $\Omega_{M}+\Omega_{\mathrm{DE}}=1$. Magnitudes quoted in the paper are corrected for Galactic extinction (Schlegel et al. 1998).

\section{LENSED QUASAR SAMPLE}

Our lensed quasar sample is constructed from the SDSS DR3 spectroscopic quasar catalog (Schneider et al. 2005). The properties of the SDSS are presented in a series of technical papers. Gunn et al. (2006) describes the dedicated wide-field 2.5-m telescope. Details of the photometric survey are given in Fukugita et al. (1996), Gunn et al. (1998), Lupton et al. (1999, 2001), Hogg et al. (2001), Smith et al. (2002), Pier et al. (2003), Ivezić et al. (2004), Tucker et al. (2006), and Lupton (2007). Blanton et al. (2003) present the tiling algorithm of the spectroscopic survey. Spectroscopic quasar targets are selected according to an algorithm described in Richards et al. (2002). Details of each public data set are given in a series of data release papers (Stoughton et al. 2002; Abazajian et al. 2003, 2004, 2005; Adelman-McCarthy 2006, 2007).

The DR3 statistical lens sample contains 11 lensed quasars (see Paper II). The sample is restricted to a range of $i$-band flux ratios, which are the fluxes of the fainter images divided by those of the brighter images for double lenses (no condition on flux ratios is set for quadruple lenses), $f_{i}>10^{-0.5}$ and image separations $1^{\prime \prime}<\theta<20^{\prime \prime}$ where the completeness of the candidate selection is almost unity (see Paper I). In this paper, we apply two additional cuts to select a subsample appropriate for our dark energy study. First, we restrict the image separation range to $1^{\prime \prime}<\theta<3^{\prime \prime}$. At $\theta<3^{\prime \prime}$ lens potentials are in many cases dominated by those from individual lensing galaxies, whereas beyond $\theta=3^{\prime \prime}$ the contribution of the surrounding dark matter to lens potentials begins to become more significant (e.g., Kochanek \& White 2001; Oguri et al. 2005b; Oguri 2006). The effect of the external field can, in principle, be included in our theoretical model of lensing rates. However, it is difficult to observationally constrain the probability distribution of external fields (note that we adopt observationally determined velocity functions for our computations; see Section 3.1), indicating that it introduces additional systematics. Second, we require that the lensing galaxy be fainter than the quasar components. If the lens galaxy is too bright, it will strongly affect the colors of the quasars and the lensed quasar will not be targeted for spectroscopy (Richards et al. 2002), biasing against us discovering the lens. In addition, lens galaxy fluxes add to the brightness of the system, which could enhance the number of lenses in the flux-limited sample. These biases make theoretical predictions much more difficult and uncertain. Therefore, we require that the $i$-band point-spread function (PSF) magnitude for the quasar components, $i_{\mathrm{qso}}$, be brighter than the $i$-band magnitude of the lensing galaxy, $i_{\text {gal }}$. These two cuts remove four lensed quasars from the lens sample. Hereafter, we use 
Table 1

SDSS DR3 Quasar Lens Sample

\begin{tabular}{lccccccccc}
\hline \hline Name & $N_{\text {img }}$ & $z_{s}$ & $z_{l}$ & $i_{\text {cor }}$ & $\theta$ & Lens & Note & Ref. \\
\hline SDSS J0246-0825 & 2 & 1.685 & 0.723 & 17.77 & 1.04 & $\mathrm{E}$ & & 1,2 \\
SBS0909+523 & 2 & 1.377 & 0.83 & 16.17 & 1.11 & $\mathrm{E}$ & & 3,4 \\
SDSS J0924+0219 & 4 & 1.523 & 0.393 & 18.12 & 1.78 & $\mathrm{E}$ & & $5,6,7$ \\
SDSS J1001+5027 & 2 & 1.839 & $\ldots$ & 17.31 & 2.86 & $\mathrm{E} ?$ & & 8 \\
SDSS J1021+4913 & 2 & 1.720 & $\ldots$ & 18.97 & 1.14 & $?$ & & 9 \\
SDSS J1226-0006 & 2 & 1.125 & 0.517 & 18.23 & 1.24 & $\mathrm{E}$ & & 10,11 \\
SDSS J1335+0118 & 2 & 1.571 & 0.440 & 17.53 & 1.57 & $\mathrm{E}$ & & 12,11 \\
Q0957+561 & 2 & 1.413 & 0.36 & 16.67 & 6.17 & $\mathrm{E}$ & $\theta>3^{\prime \prime}$ & 13,14 \\
SDSS J1004+4112 & 5 & 1.740 & 0.68 & 18.84 & 14.6 & $\mathrm{C}$ & $\theta>3^{\prime \prime}$ & 15,16 \\
SDSS J1332+0347 & 2 & 1.438 & 0.191 & 17.89 & 1.14 & $\mathrm{E}$ & $i_{\text {qso }}-i_{\text {gal }}>0$ & 17 \\
SDSS J1524+4409 & 2 & 1.210 & 0.310 & 18.76 & 1.67 & $\mathrm{E}$ & $i_{\text {qso }}-i_{\text {gal }}>0$ & 18 \\
\hline
\end{tabular}

Notes. See Paper II for details of the construction of the statistical lens sample. $i_{\text {cor }}$ is the $i$-band PSF magnitude of the object with SDSS spectroscopy, corrected for Galactic extinction. The image separation $\theta$ is defined by the maximum separation between any image pairs. "Lens" indicates the morphology of the lensing galaxy: $\mathrm{S}=$ spiral; $\mathrm{E}=$ elliptical; $\mathrm{C}=$ cluster; ? = unknown. We adopt the first seven lenses for our statistical study; the four lower lenses are not used because of reasons indicated in the Note.

References. (1) Inada et al. 2005; (2) Eigenbrod et al. 2007; (3) Oscoz et al. 1997; (4) Lubin et al. 2000; (5) Inada et al. 2003a; (6) Ofek et al. 2006; (7) Eigenbrod et al. 2006a; (8) Oguri et al. 2005a; (9) Pindor et al. 2006; (10) Inada et al. 2003c; (11) Eigenbrod et al. 2006b; (12) Oguri et al. 2004b; (13) Walsh et al. 1979; (14) Young et al. 1981; (15) Inada et al. 2003b; (16) Oguri et al. 2004a; (17) Morokuma et al. 2007; (18) Oguri et al. 2008.

the remaining seven lensed quasars to constrain cosmological parameters. Table 1 summarizes the lens sample we use in this paper.

\section{THEORETICAL MODEL}

In this section, we describe how to compute the expected number of lensed quasars in the SQLS DR3 sample in a cosmological model, following Turner et al. (1984) with the selection function taken into account.

\subsection{Lens Galaxy Population}

We consider early-type galaxies as lensing objects. Although late-type galaxies are more abundant, standard models predict that the strong lensing probability is dominated by that from early-type galaxies (e.g., Turner et al. 1984; Maoz \& Rix 1993; Kochanek 1996; Möller et al. 2007). This is particularly true if we restrict image separations to be larger than $1^{\prime \prime}$, because late-type galaxies have smaller velocity dispersions on average and therefore have smaller mean image separations. Indeed this is confirmed by observations. Only a few of the $\gtrsim 60$ known lensed quasars with $\theta>1^{\prime \prime}$ are produced by late-type galaxies. ${ }^{33}$ Moreover, none of the lensed quasars in our sample appear to be caused by late-type galaxies (see Table 1).

The contribution of large-scale dark matter fluctuations around lensing galaxies (environmental convergence) is important because it could bias cosmological results from lensing statistics (Keeton \& Zabludoff 2004). The maximum image separation of $3^{\prime \prime}$, however, makes the effect of external convergence due to the associated dark matter on the lensing probability moderate. Specifically, the environmental convergence enhances the integrated lensing probability only by $\lesssim 10 \%$ at $\theta<3^{\prime \prime}$ (Oguri et al. 2005b). Therefore we neglect external convergence, although we examine its impact on our results below.

\footnotetext{
${ }^{33}$ See the CASTLES webpage at http://cfa-www.harvard.edu/castles/.
}

It has been argued that the radial mass profile of galaxies can be approximated as a singular isothermal sphere for the scales relevant for strong lensing (e.g., Rusin \& Kochanek 2005; Koopmans et al. 2006). In this paper, we adopt an elliptical version of this, a Singular Isothermal Ellipsoid (SIE). The ellipticity does not significantly affect the total lensing cross section (Huterer et al. 2005), but including ellipticities allow one to take into account of the different selection functions of double and quadruple lenses (see Paper I). The two-dimensional surface mass density of an SIE with ellipticity $e$ at a position $x$ and $y$ from the center of the galaxy, with the $x$ axis aligned with the major axis, is given by

$$
\kappa(x, y)=\frac{\theta_{\mathrm{E}} \lambda(e)}{2}\left[\frac{1-e}{(1-e)^{2} x^{2}+y^{2}}\right]^{1 / 2},
$$

where $\theta_{\mathrm{E}}$ denotes the Einstein radius, which in turn is related to the galaxy velocity dispersion $\sigma_{v}$ by

$$
\theta_{\mathrm{E}}=4 \pi\left(\frac{\sigma_{v}}{c}\right)^{2} \frac{D_{\mathrm{ls}}}{D_{\mathrm{os}}}
$$

where $D_{\mathrm{ls}}$ and $D_{\mathrm{os}}$ are the angular diameter distances from lens to source and from observer to source, respectively. The parameter $\lambda(e)$ is the velocity dispersion normalization factor for non-spherical galaxies.

It is not straightforward to determine the normalization factor $\lambda(e)$. What is needed is the calculation of velocity dispersions for lens galaxies, which depends on the three-dimensional shape of the lensing galaxies when the assumption of spherical symmetry is relaxed. Two extreme possibilities are that all galaxies have either oblate or prolate shapes. In this paper, we assume that there are equal number of oblate and prolate galaxies and compute the normalization by taking the average of normalizations in the oblate and prolate cases (see Chae 2003). This is a reasonable assumption given the fact that underlying dark halos have triaxial shapes (Jing \& Suto 2002). Moreover the observed axis ratio distribution is consistent with a population of triaxial early-type galaxies (Vincent \& Ryden 2005). For the distribution of ellipticities, we adopt a Gaussian distribution with mean $\bar{e}=0.3$ and dispersion $\sigma_{e}=0.16$ (but truncated at $e=0$ and 0.9 ) that is consistent with the observed ellipticity distributions of the light of early-type galaxies (Bender et al. 1989; Saglia et al. 1993; Jorgensen et al. 1995; Rest et al. 2001; Alam \& Ryden 2002; Sheth et al. 2003).

One of the most important elements in predicting the number of lensed quasars is the velocity function of galaxies. As a fiducial velocity function we adopt that derived from the latest SDSS DR3 data (Choi et al. 2007), which is fitted by a modified Schechter function of the form

$$
\frac{d n}{d \sigma_{v}}=\phi_{*}\left(\frac{\sigma_{v}}{\sigma_{*}}\right)^{\alpha} \exp \left[-\left(\frac{\sigma_{v}}{\sigma_{*}}\right)^{\beta}\right] \frac{\beta}{\Gamma(\alpha / \beta)} \frac{d \sigma_{v}}{\sigma_{v}},
$$

where $\left(\phi_{*}, \sigma_{*}, \alpha, \beta\right)=\left(8.0 \times 10^{-3} h^{3} \mathrm{Mpc}^{-3}, 161 \mathrm{~km} \mathrm{~s}^{-1}, 2.32\right.$, 2.67). This is somewhat different from the velocity function derived by Sheth et al. (2003) [see also Mitchell et al. 2005] upon SDSS DR1 data. They fit the same functional form, but found different parameters $\left(\phi_{*}, \sigma_{*}, \alpha, \beta\right)=\left(4.1 \times 10^{-3} h^{3} \mathrm{Mpc}^{-3}\right.$, $\left.88.8 \mathrm{~km} \mathrm{~s}^{-1}, 6.5,1.93\right)$. We will use the parameters of Sheth et al. (2003) to estimate the size of the systematic error due to the velocity function.

Since the lens galaxy will typically have $D_{\mathrm{ls}} \sim 0.5 D_{\mathrm{os}}$, the lenses in our survey can have redshifts up to $z \sim 1$ 
(e.g., SBS0909+523 has the lens redshift of 0.83), and thus any redshift evolution of the velocity function, which could change the lensing rate and degenerate with cosmology (Keeton 2002), must be taken into account. While it has been argued that early-type galaxies evolve only through passive luminosity evolution at $z \lesssim 1$ (e.g., Im et al. 2002; Willmer et al. 2006), theoretical studies favor slight evolution with redshift through mergers (e.g., Newman \& Davis 2000). On the other hand the lens redshift distribution of strong lensing is consistent with no evolution of the velocity function (Ofek et al. 2003; Chae \& Mao 2003; Mitchell et al. 2005; Capelo \& Natarajan 2007). In this paper we assume that the velocity function does not evolve, but we also consider the evolution model used in Chae (2007) (based on a semi-analytic model by Kang et al. 2005) as well to estimate the systematic impact of the evolution on our result. In the model the number density and the characteristic velocity dispersion are simply replaced by $\phi_{*} \rightarrow \phi_{*}(1+z)^{-0.229}$ and $\sigma_{*} \rightarrow \sigma_{*}(1+z)^{-0.01}$.

\subsection{Quasar Luminosity Function}

The quasar luminosity function is used to calculate the magnification bias. We adopt the luminosity function constrained from the combination of the SDSS and $2 \mathrm{dF}$ (2SLAQ), namely the 2SLAQ+Croom et al. (2004) model in Richards et al. (2005), as our fiducial model:

$$
\begin{gathered}
\Phi\left(M_{g}\right)=\frac{\Phi_{*}}{10^{0.4\left(1-\beta_{\mathrm{h}}\right)\left(M_{g}-M_{g}^{*}\right)}+10^{0.4\left(1-\beta_{1}\right)\left(M_{g}-M_{g}^{*}\right)}}, \\
M_{g}^{*}(z)=M_{g}^{*}(0)-2.5\left(k_{1} z+k_{2} z^{2}\right),
\end{gathered}
$$

with the parameters of $\left(\beta_{\mathrm{h}}, \beta_{1}, \Phi_{*}, M_{g}^{*}(0), k_{1}, k_{2}\right)=$ $\left(3.31,1.45,1.83 \times 10^{-6}(h / 0.7)^{3} \mathrm{Mpc}^{-3} \mathrm{mag}^{-1},-21.61+\right.$ $5 \log (h / 0.7), 1.39,-0.29)$. The bright and faint end slopes are broadly consistent with those in the bolometric luminosity function of Hopkins et al. (2007) at $z \sim 1-2$. The luminosity function is in terms of rest-frame $g$-band absolute magnitudes: we convert it to observed $i$-band apparent magnitudes using the $\mathrm{K}$-correction derived in Richards et al. (2006). Since the luminosity function was derived assuming $\Omega_{M}=0.3$ and $\Omega_{\Lambda}=0.7$, we adopt this cosmology for computing the absolute magnitudes used to compute the magnification bias no matter what values of $\Omega_{\mathrm{M}}$ and $w$ we consider for the remainder of the analysis.

\subsection{Number of Lensed Quasars}

The lensing cross section $\sigma_{\text {lens }}$ for a given lens is computed numerically using the public code lensmodel (Keeton 2001). We compute the sum:

$$
\sigma_{\text {lens }, i}=\int d \mathbf{u} \frac{\Phi(L / \mu)}{\mu \Phi(L)},
$$

over the source plane positions $\mathbf{u}$ (with magnification $\mu$ ) where multiple images are produced. The cross sections are computed in units of $\theta_{\mathrm{E}}$ and they are weighted by the ratio of the differential luminosity functions in order to take magnification bias into account. The subscript $i$ indicates the number of images, with $i=2$ for double lenses and $i=4$ for quadruple lenses. For double lenses the integral is performed over the region where the flux ratio of faint to bright images is larger than $10^{-0.5}$, in order to match the selection function of our lensed quasar sample. In Paper I, we found that the magnification factor of lensed images depends on the image separation, because the
SDSS quasar target selection adopts the PSF magnitude for the magnitude limit. It was found that the dependence is fitted well by the following form (see Paper I for details):

$$
\mu=\bar{\mu} \mu_{\mathrm{tot}}+(1-\bar{\mu}) \mu_{\mathrm{bri}},
$$

and

$$
\bar{\mu}=\frac{1}{2}[1+\tanh (1.76-1.78 \theta)],
$$

where $\theta$ is in units of arcsecond and $\mu_{\text {tot }}$ and $\mu_{\text {bri }}$ are the total magnification and magnification of the brightest image, respectively. ${ }^{34}$ We compute the lensing cross section as a function of dimensionless image separation $\hat{\theta}=\theta / \theta_{\mathrm{E}}$, i.e., $d \sigma_{\text {lens }, i} / d \hat{\theta}$.

From the lensing cross section, we can compute the differential probability that a source at $z=z_{s}$ is strongly lensed with the image separation $\theta$ as

$$
\begin{aligned}
& \frac{d p_{i}}{d \theta}\left(z_{s}, i_{\mathrm{qso}}\right)=\phi_{i}(\theta) \int_{0}^{z_{s}} d z_{l} \frac{c d t}{d z_{l}}\left(1+z_{l}\right)^{3} \\
& \quad \times \int d \sigma_{v} \frac{d n}{d \sigma_{v}} \int d \hat{\theta}\left(D_{\mathrm{ol}} \theta_{\mathrm{E}}\right)^{2} \frac{d \sigma_{\mathrm{lens}, i}}{d \hat{\theta}} \\
& \quad \times \delta\left(\theta_{\mathrm{E}} \hat{\theta}-\theta\right) \Theta\left(i_{\mathrm{gal}}-i_{\mathrm{qso}}\right) \\
& =\phi_{i}(\theta) \int_{0}^{z_{s}} d z_{l} \frac{c d t}{d z_{l}}\left(1+z_{l}\right)^{3} \\
& \quad \times \int \frac{d \hat{\theta}}{\hat{\theta}} \frac{d \sigma_{v}}{d \theta_{\mathrm{E}}} \frac{d n}{d \sigma_{v}}\left(D_{\mathrm{ol}} \theta_{\mathrm{E}}\right)^{2} \frac{d \sigma_{\text {lens }, i}}{d \hat{\theta}} \Theta\left(i_{\mathrm{gal}}-i_{\mathrm{qso}}\right),
\end{aligned}
$$

where $\phi_{i}(\theta)$ is the completeness of our lens candidate selection estimated from simulations of the SDSS images (see Paper I; for double lenses we adopt the completeness averaged over the flux ratio between $10^{-0.5}$ and 1$), c d t / d z_{l}$ denotes the proper differential distance at $z_{l}$, and $\left(D_{\mathrm{ol}} \theta_{\mathrm{E}}\right)^{2}$ converts the lensing cross section from the dimensionless unit to the physical unit. The Heaviside step function $\Theta\left(i_{\text {gal }}-i_{\text {qso }}\right)$ is added to include the condition that the quasar components should be brighter than the lensing galaxy. We compute $i_{\text {gal }}$ using the correlation between the luminosity and velocity dispersion of early-type galaxies measured by Bernardi et al. (2003) combined with K-correction from Fukugita et al. (1995). The effect of the luminosity evolution measured by Bernardi et al. (2003) is included. The estimated galaxy magnitudes are broadly consistent with those expected from the empirical scaling observed for known lenses (Rusin et al. 2003). The total lensing probability for image separations between $1^{\prime \prime}$ and $3^{\prime \prime}$ is then given by

$$
p_{i}\left(z_{s}, i_{\mathrm{qso}}\right)=\int_{1^{\prime \prime}}^{3^{\prime \prime}} d \theta \frac{d p_{i}}{d \theta}\left(z_{s}, i_{\mathrm{qso}}\right) .
$$

The expected number of lensed quasars in our quasar sample is computed by counting the number of quasars weighted by the lensing probability. Ultimately this can be done by adding the probabilities for all source quasars:

$$
N_{i}=\sum_{\text {source QSOs }} p_{i}\left(z_{s}, i_{\mathrm{qso}}\right) .
$$

To save computational time, we actually calculate the expected number of lensed quasars for each redshift-magnitude bin and

\footnotetext{
34 We note that Equation (15) of Paper I holds only approximately if the slope of the source luminosity function is close to -2 . In this paper we compute the full magnification bias using Equations (6) and (7).
} 
then sum over the bins. If $N_{\mathrm{qso}}\left(z_{s, j}, i_{\mathrm{qso}, k}\right)$ is the number of source quasars in the redshift range $z_{s, j}-\Delta z_{s} / 2<z_{s}<z_{s, j}+\Delta z_{s} / 2$ and a magnitude range $i_{\mathrm{qso}, k}-\Delta i / 2<i_{\mathrm{qso}}<i_{\mathrm{qso}, k}+\Delta i / 2$, then the predicted number of lensed quasars is

$$
N_{i}=\sum_{z_{s, j}} \sum_{i_{\mathrm{qso}, k}} N_{\mathrm{qso}}\left(z_{s, j}, i_{\mathrm{qso}, k}\right) p_{i}\left(z_{s, j}, i_{\mathrm{qso}, k}\right) .
$$

We adopt bin widths of $\Delta z_{s}=0.1$ and $\Delta i=0.2$.

\subsection{Likelihood}

We perform a likelihood analysis on the DR3 SQLS lens sample. The likelihood is computed using the method introduced by Kochanek (1993):

$$
\begin{aligned}
\ln L & =\ln \left[\prod_{\text {lens }} \frac{d p_{i}}{d \theta} \prod_{\text {unlensed QSOs }}\left(1-p_{2}-p_{4}\right)\right] \\
& \simeq \sum_{\text {lens }} \ln \left(\frac{d p_{i}}{d \theta}\right)-\left(N_{2}+N_{4}\right),
\end{aligned}
$$

where $d p_{i} / d \theta$ is calculated from Equation (9) and $N_{2}$ (doubles) and $N_{4}$ (quadruples) are from Equation (12). We note that the valid approximation $p_{2}, p_{4} \ll 1$ is used here. We neglect three image events caused by naked cusps because they make a negligible contribution to the total cross section. The summation in the first term runs over the seven lensed quasars listed in Table 1. The distribution of lens redshifts offers an independent test of cosmological model; however it has been shown that it is more sensitive to the redshift evolution of the lens galaxy population as well as the selection bias (e.g., Ofek et al. 2003; Capelo \& Natarajan 2007). We do not include information on the lens redshift $z_{l}$ (i.e., we adopt the probability after integrating over the lens redshift in Equation (9)) because the lens redshifts are not known for all lenses (see Table 1) and including $z_{l}$ for those objects that do have lens redshifts might introduce systematic effect related to the incompleteness of our redshift information.

\section{RESULTS}

In this section, we derive constraints on cosmological parameters from the lens statistics. Since the abundance of lensing galaxies we adopt in this paper was determined observationally, our results do not depend on the normalization $\sigma_{8}$ or shape of the primordial power spectrum. The Hubble constant is not important for these calculation because no absolute length scale is used. We assume a flat universe $\Omega_{M}+\Omega_{\mathrm{DE}}=1$, therefore there are only two independent cosmological parameters in our analysis, $\Omega_{\mathrm{DE}}\left(\Omega_{\Lambda}\right)$ and $w$.

\subsection{Cosmological Constant}

First we derive constraints on the cosmological constant $\Omega_{\Lambda}$ assuming $w=-1$. We compute the likelihood (Equation (13)) as a function of $\Omega_{\Lambda}$, which is plotted in Figure 1. The resulting constraint $\Omega_{\Lambda}=0.74_{-0.15}^{+0.11}(1 \sigma$; the error is estimated from $\left.\Delta \chi^{2} \equiv-2 \ln \left(L / L_{\max }\right)=1\right)$ is broadly consistent with other measurements of the cosmological constant (e.g., Spergel et al. 2007, and references therein). It is also consistent with CLASS, for which the best-fit cosmological constant was $\Omega_{\Lambda}=0.7-0.8$ (Chae 2003, 2007; Mitchell et al. 2005). The case $\Omega_{\Lambda}=0$ has $\Delta \chi^{2} \sim 9$ (the expected total number of lenses in the DR3 sample is $\sim 1$ ) and is therefore rejected at the $3 \sigma$ level.

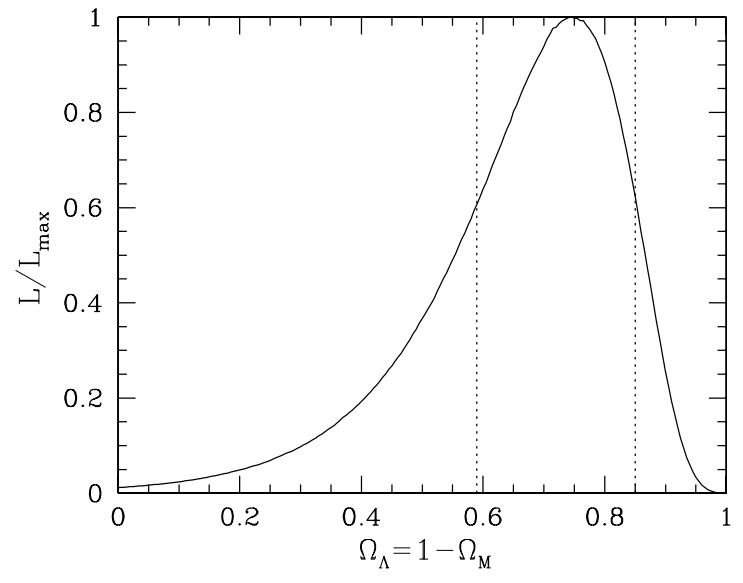

Figure 1. Relative likelihoods of the value of the cosmological constant $\Omega_{\Lambda}$ from fitting the SQLS DR3 data, assuming a spatially flat universe. The vertical dotted lines indicate the $1 \sigma$ range estimated from $\Delta \chi^{2}=1$. The likelihood becomes maximum at $\Omega_{\Lambda}=0.74$.

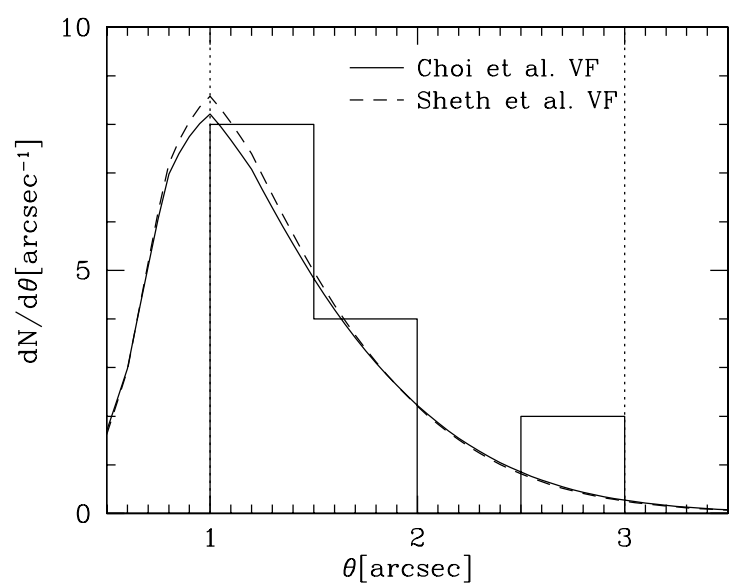

Figure 2. The number distribution of lensed quasars is plotted as a function of the image separation $\theta$. The histogram shows the number distribution in the SQLS DR3 statistical sample (see Table 1. The bin size is 0".5, thus the actual number of lenses in each bin is half of what we plot). We use only lenses in the image separation range $1^{\prime \prime}<\theta<3^{\prime \prime}$ as indicated by the vertical dotted lines. The solid line indicates the prediction of our best-fit model $\Omega_{\Lambda}=0.74$ (see Figure 1). The dashed line shows the prediction of our best-fit model when we adopt the velocity function of Sheth et al. (2003) instead of our fiducial velocity function of Choi et al. (2007). See Section 4.3 for a detailed discussion of the effect of adopting the different velocity functions. The sharp decline below $\theta=1^{\prime \prime}$ is due to the selection function $\phi_{i}(\theta)$, which rapidly decreases at $\theta<1^{\prime \prime}$. Note that our statistical lens sample contains two more lensed quasars at $\theta>3^{\prime \prime}$ that are not shown in this figure.

We test the validity of our best-fit model $\left(\Omega_{\Lambda}=0.74\right)$ by comparing the image separation distribution with the observation. In Figure 2 we plot the expected number distribution (Equation (9) summed over all sources) with the image separations of seven lensed quasars in the statistical sample. Although the small number of lensed quasars prevents detailed comparison, both the normalization and the overall shape of the curve appear to match the observed one. The Kolmogorov-Smirnov test finds that the best-fit model is consistent with the observed distribution at a significance level of $72 \%$.

\subsection{Dark Energy}

Next we derive constraints on the equation of state $w$ as well as the dark energy abundance $\Omega_{\mathrm{DE}}$. To do so we compute 


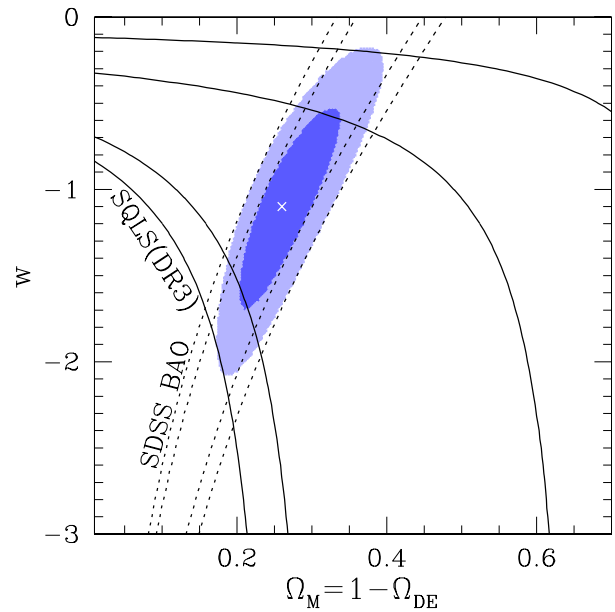

Figure 3. Contours at $1 \sigma$ and $2 \sigma$ confidence levels (estimated from $\Delta \chi^{2}=2.3$ and 6.17) are plotted in the $\Omega_{\mathrm{M}}-w$ plane. Solid lines indicate the constraint from the SQLS DR3, whereas dotted lines are from the baryon acoustic oscillations (BAO) detected in the SDSS luminous red galaxy power spectrum (Eisenstein et al. 2005). The joint constraint from SQLS and BAO is shown by shaded regions: the best-fit model $\left(\Omega_{\mathrm{M}}, w\right)=(0.26,-1.1)$ is indicated with a cross.

(A color version of this figure is available in the online journal)

the likelihood as a function of $\Omega_{\mathrm{M}}=1-\Omega_{\mathrm{DE}}$ and $w$, and compute constraints in the two-parameter space. The result is shown in Figure 3. The confidence region shows degeneracy in a similar direction as constraints from type-Ia supernovae (e.g., Astier et al. 2006; Wood-Vasey et al. 2007), therefore strong lensing alone does not constrain these parameters very well. As a complementary constraint, we also consider the likelihood from the measurement of the scale of baryon acoustic oscillations (BAO) in the SDSS luminous red galaxy power spectrum (Eisenstein et al. 2005). Specifically we adopt their constraint $A \equiv D_{V}(0.35) \sqrt{\Omega_{M} H_{0}^{2}} / 0.35 c=0.469 \pm 0.017$, where $D_{V}(0.35)$ is the dilation scale at $z=0.35$. From the combined constraint shown in Figure 3 , we obtain $\Omega_{\mathrm{M}}=$ $0.26_{-0.06}^{+0.07}$ and $w=-1.1 \pm 0.6(1 \sigma)$. Again the constraint is consistent with other measurements of dark energy (e.g., Tegmark et al. 2006; Astier et al. 2006; Spergel et al. 2007; Wood-Vasey et al. 2007).

\subsection{Systematic Errors}

In this paper we have made a number of assumptions to compute the expected number of lensed quasars in each cosmological model. Here we examine the sensitivity of our results to these assumptions. To do so, we change the input model within the expected uncertainties, and compute the bestfit cosmological parameters to determine their sensitivity to these details. We consider the following sources of systematic errors.

1. For the mass distribution of the lens galaxy, we change the fraction of prolate/oblate shapes by $\pm 25 \%$ to derive a rough estimate of the systematic error coming from the dynamical normalization. We also change the peak of the ellipticity distribution by \pm 0.1 , roughly corresponding to the current uncertainty in observations (Bender et al. 1989; Saglia et al. 1993; Jorgensen et al. 1995; Rest et al. 2001; Alam \& Ryden 2002; Sheth et al. 2003).

2. We change the faint-end slope of the quasar luminosity function by \pm 0.2 while keeping the other parameters fixed,
Table 2

Systematic Errors

\begin{tabular}{lcccc}
\hline \hline Uncertainty & $w=-1$ & & \multicolumn{2}{c}{$w \neq-1$ (with BAO) } \\
\cline { 2 - 3 } & $\Delta \Omega_{\Lambda}$ & & $\Delta \Omega_{\mathrm{M}}$ & $\Delta w$ \\
\hline Prolate 25\%-75\% & \pm 0.04 & & \pm 0.02 & ${ }_{-0.2}$ \\
$\bar{e} \rightarrow \pm 0.1$ & -0.01 & & +0.00 & +0.0 \\
$\beta_{l} \rightarrow \pm 0.2$ & \pm 0.04 & & \pm 0.02 & \pm 0.2 \\
External shear & +0.00 & -0.00 & -0.0 \\
External convergence & -0.01 & +0.01 & +0.1 \\
Sheth et al. $d n / d \sigma_{v}$ & +0.10 & -0.04 & -0.4 \\
$d n / d \sigma_{v}$ evolution & +0.04 & -0.02 & -0.2 \\
$i_{\text {qso }}-i_{\text {gal }} \rightarrow \pm 0.5$ & ${ }_{-0.02}^{+0.04}$ & \pm 0.01 & \pm 0.1 \\
Total & ${ }_{-0.06}^{+0.13}$ & & -0.03 & -0.3 \\
\hline
\end{tabular}

Note. Total errors are estimated from the quadrature sum of all errors.

in order to examine the sensitivity to the quasar luminosity function. We experiment with the faint-end slope because the most poorly constrained part of the quasar luminosity function is the faint-end slope with some evidence that it is shallower than in our model (Jiang et al. 2006). The observed quadruple lens fraction in the SQLS also favors a slightly shallower faint-end slope (Oguri 2008).

3 . In this paper we have neglected the contribution of external convergence and shear (Oguri et al. 2005b): to see how the external fields change the predicted number of lenses we simply assume the redshift-independent distributions of external fields are $0.05 \pm 0.2$ dex for shear and $0.01 \pm 0.5$ dex for convergence (e.g., Dalal \& Watson 2004, see also Momcheva et al. 2006).

4. For the velocity function, we replace the function we use with that of Sheth et al. (2003). We also consider the effect of redshift evolution of the velocity function by using a simple parametric model (see Section 3.1 for details).

5. The condition $i_{\mathrm{qso}}-i_{\mathrm{gal}}<0$ may involve some uncertainties in estimating the galaxy luminosity or the condition itself. Thus we allow an uncertainty of \pm 0.5 in the cut to estimate the systematic error that this introduces. The lens sample is unaffected by this change.

Our results summarized in Table 2 indicate that the largest uncertainties in our conclusions come from the dynamical normalization, the faint end of the quasar luminosity functions, the velocity function of the lens galaxies and its redshift evolution. In particular, changing the velocity function significantly increases the best-fit value of the cosmological constant, as discussed by Chae (2007). In essence, the lensing optical depth scales as $\sigma_{*}^{4}$, so small errors in the velocity function lead to much larger errors in the cosmological estimates. Ideally we would self-calibrate the velocity function based on the image separations (see Kochanek 1993), but the two models we consider here have very similar predictions for the image separations (see Figure 2) and the data only favor the Choi et al. (2007) model by the $\chi^{2}$ difference of $\sim 0.5$. Our result indicates that the errors from the galaxy ellipticity and external perturbations are negligibly small. If we combine all these uncertainties, we obtain systematic errors comparable to the statistical errors, e.g., $\Omega_{\Lambda}=0.74_{-0.15}^{+0.11}(\text { stat. })_{-0.06}^{+0.13}$ (syst.) for the cosmological constant case.

\section{SUMMARY AND DISCUSSION}

We have derived constraints on dark energy using the new optical strong lens sample from the SQLS DR3. We take various 
selection effects into account to make reasonably robust predictions for the number of lensed quasars in the SQLS. We have found that the derived constraints agree well with the current concordance cosmology. Assuming a cosmological constant $(w=-1)$ we have obtained $\Omega_{\Lambda}=0.74_{-0.15}^{+0.11}$ (stat. $)_{-0.06}^{+0.13}$ (syst.). The constraint primarily arises from the total number of lenses in our statistical sample. For the more general case $w \neq-1$, the constraint was combined with that of the SDSS BAO (Eisenstein et al. 2005) to break the degeneracy. The resulting joint constraint is $\Omega_{\mathrm{M}}=0.26_{-0.06}^{+0.07}$ (stat. $)_{-0.05}^{+0.03}$ (syst.) and $w=-1.1 \pm 0.6$ (stat. $)_{-0.5}^{+0.3}$ (syst.). The results are in good agreement with recent constraints from radio lenses (Chae 2003, 2007; Mitchell et al. 2005). The results confirm the current standard picture that the universe is dominated by dark energy with a cosmological constant-like equation of state, independently of type Ia supernovae.

Although we have quantified our systematics on our cosmological results by changing several important assumptions, there are additional systematic effects that could change our quantitative results. One is dust extinction by lensing galaxies. Previous studies have shown that dust is indeed present in lensing galaxies even if they are early-type galaxies, although the measured total extinction is modest, $E(B-V) \sim 0.1 \mathrm{mag}$ (e.g., Falco et al. 1999). Since we set the magnitude limit at the $i$-band for which dust extinction is less important than in the bluer bands, we expect that this effect will not heavily influence our result. Moreover, the flux from the lensing galaxy slightly increases the PSF magnitude of the lens system, and this effect could compensate the effect of dust extinction to some extent. Another effect we have neglected is lensing by multiple galaxies. Theoretically the probability for such multiple lensing is just a few percent of the lensing probability by a single galaxy at $z \lesssim 2$ (Möller \& Blain 2001) and therefore can be neglected. However, it only considered chance superpositions along the line of sight and ignored nearby correlated galaxies that could dominate the contribution to multiple lens events (e.g., Cohn \& Kochanek 2004). Moreover, the fact that one of our lens sample, SDSS J1001+5027 (Oguri et al. 2005a), has a secondary galaxy near the primary lensing galaxy suggests that the effect needs to be addressed carefully.

In this paper we have assumed a flat universe. Although this is a reasonable assumption given that virtually all current cosmological constraints are consistent with a flat universe (e.g., Tegmark et al. 2006; Ichikawa \& Takahashi 2007; Spergel et al. 2007; Wang \& Mukherjee 2007; Allen et al. 2007), it is still of interest to consider non-flat universes. In particular the introduction of both non-flatness and $w \neq-1$ results in additional strong degeneracy between cosmological parameters (e.g., Linder 2005), and thus may require an additional independent cosmological probe in order to obtain tight constraints on individual parameters.

Cosmological constraints presented in this paper are obtained from the SQLS DR3 sample, which represents $\sim 40 \%$ of the full SDSS data. The extension of the SQLS lens sample to the SDSS DR5 and SDSS-II is in progress; therefore we expect that the statistical errors will improve significantly in the near future. Better constraints may be obtained by considering the SQLS and CLASS samples jointly. In addition to the extension of the lens sample, it is of great importance to reduce the systematic errors. An important advantage of our optical survey over radio surveys is that there is no systematics from the source redshift distribution, which was the biggest source of systematic error in the CLASS analysis (Chae 2003). The velocity function of galaxies and the faint and quasar luminosity function are expected to converge in the near future as current large-scale surveys are completed, thus we expect that the systematic errors can be reduced in future analyses of the SQLS lenses. If we have a large enough sample of lenses, we may be able to reduce the systematic effect further by calibrating the velocity function from the observed image separation distribution itself.

Although in this paper we have restricted ourselves to galaxyscale lenses to study dark energy, our statistical lens sample contains group- or cluster-scale lenses as well. The number of cluster-scale lenses in our sample is quite sensitive to the abundance of clusters at intermediate redshifts $(z \sim 0.5)$, and therefore was used to study $\sigma_{8}$ (Oguri \& Keeton 2004; Li et al. 2007). The full image separation distribution from galaxy scales to cluster scales will be valuable in understanding how galaxies are populated in dark matter halos.

We thank Kyu-Hyun Chae for useful discussions. This work was supported in part by the Department of Energy contract DEAC02-76SF00515. N.I. acknowledges supports from the Japan Society for the Promotion of Science and the Special Postdoctoral Researcher Program of RIKEN. M.A.S. acknowledges support from NSF grant AST 03-07409. A portion of this work was also performed under the auspices of the U.S. Department of Energy, National Nuclear Security Administration by the University of California, Lawrence Livermore National Laboratory under contract No. W-7405-Eng-48. I.K. acknowledges supports from Ministry of Education, Culture, Sports, Science, and Technology, Grant-in-Aid for Encouragement of Young Scientists (No. 17740139), and Grant-in-Aid for Scientific Research on Priority Areas No. 467 "Probing the Dark Energy through an Extremely Wide \& Deep Survey with Subaru Telescope". A.C. acknowledges the support of CONICYT, Chile, under grant FONDECYT 1051061.

Funding for the SDSS and SDSS-II has been provided by the Alfred P. Sloan Foundation, the Participating Institutions, the National Science Foundation, the U.S. Department of Energy, the National Aeronautics and Space Administration, the Japanese Monbukagakusho, the Max Planck Society, and the Higher Education Funding Council for England. The SDSS Web site is http://www.sdss.org/.

The SDSS is managed by the Astrophysical Research Consortium for the Participating Institutions. The Participating Institutions are the American Museum of Natural History, Astrophysical Institute Potsdam, University of Basel, Cambridge University, Case Western Reserve University, University of Chicago, Drexel University, Fermilab, the Institute for Advanced Study, the Japan Participation Group, Johns Hopkins University, the Joint Institute for Nuclear Astrophysics, the Kavli Institute for Particle Astrophysics and Cosmology, the Korean Scientist Group, the Chinese Academy of Sciences (LAMOST), Los Alamos National Laboratory, the Max-Planck-Institute for Astronomy (MPIA), the Max-Planck-Institute for Astrophysics (MPA), New Mexico State University, Ohio State University, University of Pittsburgh, University of Portsmouth, Princeton University, the United States Naval Observatory, and the University of Washington.

\section{REFERENCES}

Abazajian, K., et al. 2003, AJ, 126, 2081

Abazajian, K., et al. 2004, AJ, 128, 502

Abazajian, K., et al. 2005, AJ, 129, 1755 
Adelman-McCarthy, J. K., et al. 2006, ApJS, 162, 38

Adelman-McCarthy, J. K., et al. 2007, ApJS, 172, 634

Alam, S. M. K., \& Ryden, B. S. 2002, ApJ, 570, 610

Allen, S. W., Rapetti, D. A., Schmidt, R. W., Ebeling, H., Morris, G., \& Fabian, A. C. 2007, MNRAS submitted (arXiv:0706.0033)

Allen, S. W., Schmidt, R. W., Ebeling, H., Fabian, A. C., \& van Speybroeck, L. 2004, MNRAS, 353, 457

Astier, P., et al. 2006, A\&A, 447, 31

Bender, R., Surma, P., Doebereiner, S., Moellenhoff, C., \& Madejsky, R. 1989, A\&A, 217, 35

Bernardi, M., et al. 2003, AJ, 125, 1849

Blanton, M. R., Lin, H., Lupton, R. H., Maley, F. M., Young, N., Zehavi, I., \& Loveday, J. 2003, AJ, 125, 2276

Browne, I. W. A., et al. 2003, MNRAS, 341, 13

Capelo, P. R., \& Natarajan, P. 2007, New J. Phys. in press (arXiv:0705.3042)

Carroll, S. M., Duvvuri, V., Trodden, M., \& Turner, M. S. 2004, Phys. Rev. D, 70,043528

Chae, K.-H. 2003, MNRAS, 346, 746

Chae, K.-H. 2007, ApJ, 658, L71

Chae, K.-H., \& Mao, S. 2003, ApJ, 599, L61

Chae, K.-H., et al. 2002, Phys. Rev. Lett., 89, 151301

Chiba, M., \& Yoshii, Y. 1999, ApJ, 510, 42

Choi, Y.-Y., Park, C., \& Vogeley, M. S. 2007, ApJ, 658, 884

Cohn, J. D., \& Kochanek, C. S. 2004, ApJ, 608, 25

Cole, S., et al. 2005, MNRAS, 362, 505

Croom, S. M., Smith, R. J., Boyle, B. J., Shanks, T., Miller, L., Outram, P. J., \& Loaring, N. S. 2004, MNRAS, 349, 1397

Dalal, N., \& Watson, C. R. 2004, arXiv astro-ph/0409483

de Bernardis, P., et al. 2000, Nature, 404, 955

Eigenbrod, A., Courbin, F., Dye, S., Meylan, G., Sluse, D., Vuissoz, C., \& Magain, P. 2006a, A\&A, 451, 74

Eigenbrod, A., Courbin, F., Meylan, G., Vuissoz, C., \& Magain, P. 2006b, A\&A, 451,759

Eigenbrod, A., Courbin, F., \& Meylan, G. 2007, A\&A, 465, 51

Eisenstein, D. J., et al. 2005, ApJ, 633, 560

Falco, E. E., Kochanek, C. S., \& Munoz, J. A. 1998, ApJ, 494, 47

Falco, E. E., et al. 1999, ApJ, 523, 617

Fukugita, M., Futamase, T., \& Kasai, M. 1990, MNRAS, 246, 24

Fukugita, M., Ichikawa, T., Gunn, J. E., Doi, M., Shimasaku, K., \& Schneider, D. P. 1996, AJ, 111, 1748

Fukugita, M., Shimasaku, K., \& Ichikawa, T. 1995, PASP, 107, 945

Gunn, J. E., et al. 1998, AJ, 116, 3040

Gunn, J. E., et al. 2006, AJ, 131, 2332

Hogg, D. W., Finkbeiner, D. P., Schlegel, D. J., \& Gunn, J. E. 2001, AJ, 122,2129

Hopkins, P. F., Richards, G. T., \& Hernquist, L. 2007, ApJ, 654, 731

Huterer, D., Keeton, C. R., \& Ma, C.-P. 2005, ApJ, 624, 34

Ichikawa, K., \& Takahashi, T. 2007, JCAP, 2, 1

Im, M., et al. 2002, ApJ, 571, 136

Inada, N., et al. 2003a, AJ, 126, 666

Inada, N., et al. 2003b, Nature, 426, 810

Inada, N., et al. $2003 \mathrm{c}, \mathrm{AJ}$, submitted

Inada, N., et al. 2005, AJ, 130, 1967

Inada, N., et al. 2008, AJ, 135, 496 (Paper II)

Ivezić, Ž., et al. 2004, AN, 325, 583

Jiang, L., et al. 2006, AJ, 131, 2788

Jing, Y. P., \& Suto, Y. 2002, ApJ, 574, 538

Jorgensen, I., Franx, M., \& Kjaergaard, P. 1995, MNRAS, 273, 1097

Kang, X., Jing, Y. P., Mo, H. J., \& Börner, G. 2005, ApJ, 631, 21

Keeton, C. R. 2001, arXiv astro-ph/0102340

Keeton, C. R. 2002, ApJ, 575, L1

Keeton, C. R., \& Zabludoff, A. I. 2004, ApJ, 612, 660

Kochanek, C. S. 1993, ApJ, 419, 12

Kochanek, C. S. 1996, ApJ, 466, 638

Kochanek, C. S., \& White, M. 2001, ApJ, 559, 531

Koopmans, L. V. E., Treu, T., Bolton, A. S., Burles, S., \& Moustakas, L. A 2006, ApJ, 649, 599

Li, G. L., Mao, S., Jing, Y. P., Lin, W. P., \& Oguri, M. 2007, MNRAS, 378, 469

Linder, E. V. 2005, Astropart. Phys., 24, 391
Lubin, L. M., Fassnacht, C. D., Readhead, A. C. S., Blandford, R. D., \& Kundić, T. 2000, AJ, 119, 451

Lupton, R. 2007, AJ, submitted

Lupton, R., Gunn, J. E., Ivezić, Z., Knapp, G. R., Kent, S., \& Yasuda, N. 2001 in ASP Conf. Ser. 238, Astronomical Data Analysis Software and Systems X, ed. F. R. Harnden, Jr., F. A. Primini, \& H. E. Payne (San Francisco, CA: ASP) 269

Lupton, R. H., Gunn, J. E., \& Szalay, A. S. 1999, AJ, 118, 1406

Maoz, D. 2005, IAU Symposium, 225, 413

Maoz, D., \& Rix, H.-W. 1993, ApJ, 416, 425

Mitchell, J. L., Keeton, C. R., Frieman, J. A., \& Sheth, R. K. 2005, ApJ, 622,81

Möller, O., \& Blain, A. W. 2001, MNRAS, 327, 339

Möller, O., Kitzbichler, M., \& Natarajan, P. 2007, MNRAS in press, (arXiv astro-ph/0607032)

Momcheva, I., Williams, K., Keeton, C., \& Zabludoff, A. 2006, ApJ, 641, 169

Morokuma, T., et al. 2007, AJ, 133, 214

Myers, S. T., et al. 2003, MNRAS, 341, 1

Newman, J. A., \& Davis, M. 2000, ApJ, 534, L11

Ofek, E. O., Maoz, D., Rix, H.-W., Kochanek, C. S., \& Falco, E. E. 2006, ApJ, 641,70

Ofek, E. O., Rix, H.-W., \& Maoz, D. 2003, MNRAS, 343, 639

Oguri, M. 2006, MNRAS, 367, 1241

Oguri, M. 2007, New J. Phys. in press (arXiv:0705.4252)

Oguri, M., et al. 2004a, ApJ, 605, 78

Oguri, M., \& Keeton, C. R. 2004, ApJ, 610, 663

Oguri, M., et al. 2004b, PASJ, 56, 399

Oguri, M., et al. 2005a, ApJ, 622, 106

Oguri, M., et al. 2006, AJ, 132, 999 (Paper I)

Oguri, M., et al. 2008, AJ, 135, 520

Oguri, M., Keeton, C. R., \& Dalal, N. 2005b, MNRAS, 364, 1451

Oscoz, A., Serra-Ricart, M., Mediavilla, E., Buitrago, J., \& Goicoechea, L. J. 1997, ApJ, 491, L7

Peebles, P. J., \& Ratra, B. 2003, Rev. Mod. Phys., 75, 559

Percival, W. J., et al. 2007, ApJ, 657, 51

Perlmutter, S., et al. 1999, ApJ, 517, 565

Pier, J. R., Munn, J. A., Hindsley, R. B., Hennessy, G. S., Kent, S. M., Lupton, R. H., \& Ivezić, Ź. 2003, AJ, 125, 1559

Pindor, B., et al. 2006, AJ, 131, 41

Rapetti, D., Allen, S. W., \& Weller, J. 2005, MNRAS, 360, 555

Rassat, A., Land, K., Lahav, O., \& Abdalla, F. B. 2007, MNRAS, 377, 1085

Rest, A., van den Bosch, F. C., Jaffe, W., Tran, H., Tsvetanov, Z., Ford, H. C. Davies, J., \& Schafer, J. 2001, AJ, 121, 2431

Riess, A. G., et al. 1998, AJ, 116, 1009

Richards, G. T., et al. 2002, AJ, 123, 2945

Richards, G. T., et al. 2005, MNRAS, 360, 839

Richards, G. T., et al. 2006, AJ, 131, 2766

Rusin, D., \& Kochanek, C. S. 2005, ApJ, 623, 666

Rusin, D., et al. 2003, ApJ, 587, 143

Saglia, R. P., Bender, R., \& Dressler, A. 1993, A\&A, 279, 75

Schlegel, D. J., Finkbeiner, D. P., \& Davis, M. 1998, ApJ, 500, 525

Schneider, D. P., et al. 2005, AJ, 130, 367

Sheth, R. K., et al. 2003, ApJ, 594, 225

Smith, J. A., et al. 2002, AJ, 123, 2121

Spergel, D. N., et al. 2003, ApJS, 148, 175

Spergel, D. N., et al. 2007, ApJS, 170, 377

Stoughton, C., et al. 2002, AJ, 123, 485

Tegmark, M., et al. 2006, Phys. Rev. D, 74, 123507

Tucker, D. L., et al. 2006, AN, 327, 821

Turner, E. L. 1990, ApJ, 365, L43

Turner, E. L., Ostriker, J. P., \& Gott, J. R., III 1984, ApJ, 284, 1

Vincent, R. A., \& Ryden, B. S. 2005, ApJ, 623, 137

Walsh, D., Carswell, R. F., \& Weymann, R. J. 1979, Nature, 279, 381

Wang, Y., \& Mukherjee, P. 2007, arXiv astro-ph/0703780

Willmer, C. N. A., et al. 2006, ApJ, 647, 853

Wood-Vasey, W. M., et al. 2007, ApJ, 666, 694

York, D. G., et al. 2000, AJ, 120, 1579

Young, P., Gunn, J. E., Oke, J. B., Westphal, J. A., \& Kristian, J. 1981, ApJ, 244,736 